MITSUBISHI ELECTRIC RESEARCH LABORATORIES

http://www.merl.com

\title{
Capacity Estimation for Lithium-ion Batteries Using Adaptive Filter on Affine Space
}

\author{
Wada, T.; Takegami, T.; Wang, Y.; Sahinoglu, Z.
}

TR2015-071 May 2015

\begin{abstract}
In this paper, we propose methods to estimate the full charge capacity (FCC) of a battery based on adaptive filters. The FCC is estimated as a ratio of the accumulated chargecurrent to the state of charge ( $\mathrm{SoC}$ ) of the battery, which is estimated by an extended Kalman filter. We consider bias errors on the estimated SoC caused by the error of typical value of FCC, which is assumed in the SoC estimation. We also consider the current sensor offset, which causes unboundedness of variables in the FCC estimation. We compose the adaptive filters on an affine space to avoid the unboundedness, which is undesirable for an implementation in embedded systems.
\end{abstract}

Asian Control Conference 2015

\begin{abstract}
This work may not be copied or reproduced in whole or in part for any commercial purpose. Permission to copy in whole or in part without payment of fee is granted for nonprofit educational and research purposes provided that all such whole or partial copies include the following: a notice that such copying is by permission of Mitsubishi Electric Research Laboratories, Inc.; an acknowledgment of the authors and individual contributions to the work; and all applicable portions of the copyright notice. Copying, reproduction, or republishing for any other purpose shall require a license with payment of fee to Mitsubishi Electric Research Laboratories, Inc. All rights reserved.
\end{abstract}





\section{Capacity Estimation for Lithium-ion Batteries Using Adaptive Filter on Affine Space}

\author{
Toshihiro Wada and Tomoki Takegami \\ Mitsubishi Electric Corporation, Advanced Technology R\&D Center \\ 8-1-1, Tsukaguchi-Honmachi \\ Amagasaki, Hyogo, 661-8661, Japan \\ Email: Wada.Toshihiro@bx.MitsubishiElectric.co.jp \\ Takegami.Tomoki@bx.MitsubishiElectric.co.jp
}

\author{
Yebin Wang and Zafer Sahinoglu \\ Mitsubishi Electric Research Laboratories \\ 201 Broadway, 8th Floor \\ Cambridge, MA 02139-1955, USA \\ Email: yebinwang@merl.com \\ zafer@merl.com
}

\begin{abstract}
In this paper, we propose methods to estimate the full charge capacity (FCC) of a battery based on adaptive filters. The FCC is estimated as a ratio of the accumulated charge current to the state of charge (SoC) of the battery, which is estimated by an extended Kalman filter. We consider bias errors on the estimated SoC caused by the error of typical value of FCC, which is assumed in the $\mathrm{SoC}$ estimation. We also consider the current sensor offset, which causes unboundedness of variables in the FCC estimation. We compose the adaptive filters on an affine space to avoid the unboundedness, which is undesirable for an implementation in embedded systems.
\end{abstract}

\section{INTRODUCTION}

Lithium-ion batteries (LiBs) have been widely used in electric appliances and electric cars; the application of LiBs becomes wider because of its high energy density, high power density and long life [1]. While it is important to assess the battery lifetime to use LiBs for larger scale applications, such as peak shaving, photovoltaic power generation and so on, the battery lifetime is however difficult to predict because of limited measurability relative to the complexity of the internal processes.

For designing battery systems, the battery lifetime is commonly assessed by its full charge capacity (FCC), although it should be evaluated by its energy storage capacity. Because the energy storage capacity depends on the internal resistance of the battery, which substantially varies depending on the temperature [2]. In addition, FCC is one of the most basic battery model parameter for the state of charge (SoC) estimation, where $\mathrm{SoC}$ is obtained as a ratio of the accumulated charge current to the FCC in Coulomb counting and several model-based methods [3]-[6].

The FCC has an initial variation in each battery cell, and decreases due to the degradation. But it takes a long time for an actual measurement of the FCC according to its definition: the electric quantity to charge the battery full from the empty. Worse yet, the battery system has to be suspended during the measurement. A solution of this problem is applying a signal processing technology. An online FCC estimation method based on an adaptive filter is proposed in [7], and another method based on Kalman filter is proposed in [8]. Although additive Gaussian noises on the terminal voltage and the current measurements are taken into consideration in these methods, a significant degradation in the estimation accuracy is caused by an offset on the current measurements, which are inevitable in widely used Hall effect sensors [9].

We consider a simultaneous estimation of the FCC and the current sensor offset by an adaptive filter. Let $q_{\mathrm{cc}, k}$ be the Coulomb counting calculated recursively by the following algorithm:

$$
q_{\mathrm{cc}, k}=q_{\mathrm{cc}, k-1}+t_{s} I_{k-1}, \quad q_{\mathrm{cc}, 0}=0
$$

where $t_{s}$ is the sampling period, $I_{k}$ is the measured current, the FCC is able to be estimated based on the following relationship:

$$
q_{\mathrm{cc}, k}=F_{\mathrm{cc}} s_{k}+t_{k} I_{\mathrm{off}}-q_{0},
$$

where $t_{k}:=k t_{s}, I_{\mathrm{off}}$ is the current sensor offset, $F_{\mathrm{cc}}$ is the FCC, $s_{k}$ is the SoC and $q_{0}$ is the initial electric quantity charged in the battery. It is able to implement a recursive least squares (RLS) filter or a recursive total least squares (RTLS) filter to estimate the $I_{\text {off }}, F_{\mathrm{cc}}$ and $q_{0}$, if an accurate estimation of $s_{k}$ is obtained [7].

The first difficulty in the FCC estimation based on the adaptive filters is that the model-based methods for SoC estimation depend on the FCC. If a typical value of the FCC is used in these methods, bias errors of $s_{k}$ are caused by the difference between the typical value and the true value of the FCC. The second difficulty is caused by the term $t_{k} I_{\text {off }}$ in (2), which increases unlimitedly with time. It is obviously undesirable for an implementation in embedded systems.

In this paper, we propose methods to estimate the FCC of a battery and the current sensor offset based on an RLS filter and an RTLS filter proposed in [10], in which the bias errors of the estimated $\mathrm{SoC}$ is compensated by a first order evaluation thereof. All variables in our methods are numerically bounded by composing the adaptive filters on an affine space.

\section{Model BASEd SoC estimation}

\section{A. Lithium-ion battery}

An LiB consists mainly of a positive electrode, a negative electrode, current collectors and a separator; all components are soaked with electrolyte solution (Fig. 1). In a typical design, the positive electrode is made of a porous material composed of metal oxide particles such as $\mathrm{LiCoO}_{2}, \mathrm{LiMn}_{2} \mathrm{O}_{4}$ and so on. The negative electrode is also made of a porous 


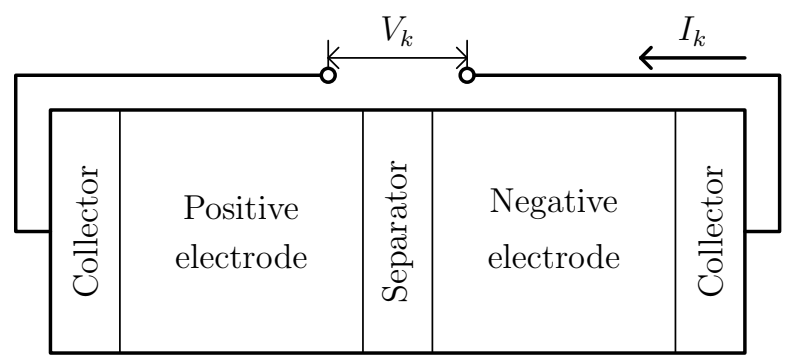

Fig. 1. A typical structure of lithium-ion battery cells. Lithium-ions pass through the separator, while electrons conduct via the electric circuit.

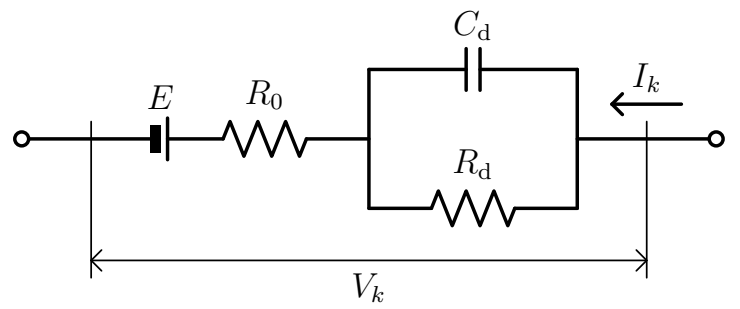

Fig. 2. An equivalent circuit expression of a simplified lithium-ion battery model. The voltage source $E$ depends on the state of charge of the battery.

material composed of graphite $\left(\mathrm{C}_{6}\right)$. Electrolyte solution is an organic solvent with electrolyte such as $\mathrm{LiPF}_{6}$ [11].

A charge-discharge reaction in the positive electrode is expressed by:

$$
\mathrm{LiCoO}_{2} \underset{\text { Discharge }}{\stackrel{\text { Charge }}{\rightleftharpoons}} \mathrm{Li}_{1-x_{\mathrm{p}}} \mathrm{CoO}_{2}+\mathrm{Li}_{x_{\mathrm{p}}}^{+}+\mathrm{e}_{x_{\mathrm{p}}}^{-}
$$

where $x_{\mathrm{p}}$ denotes the number of reaction electrons. In the negative electrode, the reaction is expressed by:

$$
\mathrm{C}_{6}+\mathrm{Li}_{x_{\mathrm{n}}}^{+}+\mathrm{e}_{x_{\mathrm{n}}}^{-} \underset{\text { Discharge }}{\stackrel{\text { Charge }}{\rightleftharpoons}} \mathrm{Li}_{x_{\mathrm{n}}} \mathrm{C}_{6}
$$

where $x_{\mathrm{n}}$ denotes the number of reaction electrons [12].

In a charge process, $\mathrm{Li}^{+} \mathrm{s}$ are emitted from the positive electrode, then absorbed into the negative electrode from the electrolyte solution. The electrons flow along the external electric circuit via the current collectors, because the electrodes are electrically isolated by the separator.

The SoC of the battery is defined as:

$$
\frac{x_{\mathrm{p}}-x_{\mathrm{p}}^{-}}{x_{\mathrm{p}}^{+}-x_{\mathrm{p}}^{-}} \text {or } \frac{x_{\mathrm{n}}-x_{\mathrm{n}}^{-}}{x_{\mathrm{n}}^{+}-x_{\mathrm{n}}^{-}}
$$

where $\left[x_{\mathrm{p}}^{-}, x_{\mathrm{p}}^{+}\right]$and $\left[x_{\mathrm{n}}^{-}, x_{\mathrm{n}}^{+}\right]$denote the rated ranges of use of the positive electrode and the negative electrode respectively.

\section{B. Electric characteristics}

Although the mathematical model of LiBs is too complex [13], we consider a simplified battery model described in [14] and an extended Kalman filter based on the model for SoC estimation to describe the evaluation of bias errors on the estimated SoC.

Fig. 2 shows an equivalent circuit model of an $\mathrm{LiB}$, where $R_{\mathrm{d}}$ and $R_{0}$ are resistors, and $C_{\mathrm{d}}$ is a capacitor. The voltage source $E$ is referred to as an open circuit voltage (OCV) which is a function of the SoC [15]. Let $q_{\mathrm{b}, k}$ and $q_{\mathrm{d}, k}$ be the electric quantities charged in the battery and the capacitor respectively, and let $\tau_{\mathrm{d}}:=R_{\mathrm{d}} C_{\mathrm{d}}$, the terminal voltage of the battery $V_{k}$ is described as follows:

$$
\boldsymbol{x}_{k+1}=\boldsymbol{F} \boldsymbol{x}_{k}+\boldsymbol{G} I_{k}, \quad V=h\left(\boldsymbol{x}_{k}\right)+R_{0} I_{k},
$$

where $\boldsymbol{x}_{k}:=\left[\begin{array}{ll}q_{\mathrm{d}, k} & q_{\mathrm{b}, k}\end{array}\right]^{\top}, h\left(\boldsymbol{x}_{k}\right):=E\left(q_{\mathrm{b}, k} / F_{\mathrm{cc}}\right)$ and

$$
\boldsymbol{F}:=\left[\begin{array}{ll}
e^{-\frac{t_{s}}{\tau_{\mathrm{d}}}} & \\
& 1
\end{array}\right], \quad \boldsymbol{G}:=\left[\begin{array}{c}
\tau_{\mathrm{d}}\left(1-e^{-\frac{t_{s}}{\tau_{\mathrm{d}}}}\right) \\
t_{s}
\end{array}\right] .
$$

\section{Extended Kalman filter for SoC estimation}

Let $\sigma_{I}^{2}$ and $\sigma_{V}^{2}$ be the variances of the noises on the current and the voltage measurement respectively, A prediction step of an extended Kalman filter (EKF) for SoC estimation is written as follows:

$$
\begin{aligned}
& \hat{\boldsymbol{x}}_{k+1 \mid k}=\boldsymbol{F} \hat{\boldsymbol{x}}_{k \mid k}+\boldsymbol{G} I_{k}, \\
& \hat{\boldsymbol{P}}_{k+1 \mid k}=\boldsymbol{F} \hat{\boldsymbol{P}}_{k \mid k} \boldsymbol{F}^{\top}+\boldsymbol{Q},
\end{aligned}
$$

where $\hat{\boldsymbol{x}}_{l \mid k}$ is an estimation of $\boldsymbol{x}_{l}$ at $t_{k}$, and $\boldsymbol{Q}$ is a symmetric positive definite matrix depending on $\sigma_{V}$. An update step of the EKF is written as follows:

$$
\begin{aligned}
& \hat{\boldsymbol{x}}_{k \mid k}=\hat{\boldsymbol{x}}_{k \mid k-1}+\boldsymbol{K}_{k}\left(V_{k}-z_{k}\right), \\
& \hat{\boldsymbol{P}}_{k \mid k}=\left(I-\boldsymbol{K}_{k} \boldsymbol{H}_{k}\right) \hat{\boldsymbol{P}}_{k \mid k-1},
\end{aligned}
$$

where

$$
\begin{aligned}
& z_{k}:=h\left(\hat{\boldsymbol{x}}_{k \mid k-1}\right)+R_{0} I_{k}, \quad \boldsymbol{H}_{k}:=\frac{\partial h}{\partial \boldsymbol{x}_{k}}\left(\hat{\boldsymbol{x}}_{k \mid k-1}\right), \\
& \boldsymbol{S}_{k}:=\sigma_{V}^{2}+\boldsymbol{H}_{k} \hat{\boldsymbol{P}}_{k \mid k-1} \boldsymbol{H}_{k}^{\top}, \quad \boldsymbol{K}_{k}:=\hat{\boldsymbol{P}}_{k \mid k-1} \boldsymbol{H}_{k}^{\top} \boldsymbol{S}_{k}^{-1} .
\end{aligned}
$$

Then the estimation of the SoC is given by $\hat{s}_{k}:=\hat{q}_{\mathrm{b}, k \mid k} / F_{\mathrm{cc}}$, where $\left[\begin{array}{ll}\hat{q}_{\mathrm{d}, k \mid k} & \hat{q}_{\mathrm{b}, k \mid k}\end{array}\right]:=\hat{\boldsymbol{x}}_{k \mid k}^{\top}$.

\section{FCC AND CURRENT SENSOR OFFSET ESTIMATION}

\section{A. Bias error compensation}

The SoC of the battery is estimated from a time series of the measured current and the terminal voltage. As we described in section II-C, the estimation algorithm depends on the FCC of the battery. Therefore the dependency of the estimation is expressed by:

$$
\hat{s}_{k}=\mathcal{F}_{k}\left(\left(I_{k}, V_{k}\right), \ldots,\left(I_{0}, V_{0}\right) \mid F_{\mathrm{cc}}\right),
$$

where $\mathcal{F}_{k}$ is a map from a set of measured values to an estimated value of SoC. If a typical value of the FCC used in the SoC estimation is slightly different from the true value of the FCC, and/or the current sensor offset, the estimated value of $\mathrm{SoC}$ is biased as expressed by:

$$
\tilde{s}_{k}=\mathcal{F}_{k}\left(\left(I_{k}+I_{\text {off }}, V_{k}\right), \ldots,\left(I_{0}+I_{\text {off }}, V_{0}\right) \mid \tilde{F}_{\mathrm{cc}}\right) .
$$

Let the current sensor offset and difference between $F_{\text {cc }}$ and $\tilde{F}_{\text {cc }}$ parameterize by $I_{\text {off }} \approx I_{\text {typ }} p_{1}$ and $\tilde{F}_{\mathrm{cc}} \approx F_{\text {cc }}\left(1+p_{2}\right)$, the biased estimation $\tilde{s}_{k}$ is approximated as:

$$
\tilde{s}_{k} \approx \hat{s}_{k}+\frac{\partial \mathcal{F}_{k}}{\partial p_{1}} p_{1}+\frac{\partial \mathcal{F}_{k}}{\partial p_{2}} p_{2}
$$


by a Taylor series expansion (see [16] for matrix derivatives), where $I_{\text {typ }}$ is a constant introduced to ensure $p_{1} \ll 1$. Then we get the following relation:

$$
\begin{array}{r}
q_{\mathrm{cc}, k}=\frac{\tilde{F}_{\mathrm{cc}}}{1+p_{2}}\left(\tilde{s}_{k}-\frac{\partial \mathcal{F}_{k}}{\partial p_{1}} p_{1}-\frac{\partial \mathcal{F}_{k}}{\partial p_{2}} p_{2}\right) \\
+I_{\mathrm{typ}} p_{1} t_{k}-q_{0}
\end{array}
$$

by substituting (13) into (2). The equation (14) is rewritten as:

$$
\begin{aligned}
q_{\mathrm{cc}, k}-\tilde{F}_{\mathrm{cc}} \tilde{s}_{k}= & \left(I_{\mathrm{typ}} t_{k}-\tilde{F}_{\mathrm{cc}} \frac{\partial \mathcal{F}_{k}}{\partial p_{1}}\right) p_{1} \\
& -\tilde{F}_{\mathrm{cc}}\left(\tilde{s}_{k}+\frac{\partial \mathcal{F}_{k}}{\partial p_{2}}\right) p_{2}-q_{0}
\end{aligned}
$$

by omitting higher order terms of $p_{1}$ and $p_{2}$.

\section{B. Adaptive filter on affine space}

For simplicity, we rewrite (15) as:

$$
y_{k}=\boldsymbol{p}^{\top} \boldsymbol{u}_{k}-q_{0},
$$

where

$$
y_{k}:=q_{\mathrm{cc}, k}-\tilde{F}_{\mathrm{cc}} \tilde{s}_{k}, \quad \boldsymbol{u}_{k}:=\left[\begin{array}{c}
I_{\mathrm{typ}} t_{k}-\tilde{F}_{\mathrm{cc}} \frac{\partial \mathcal{F}_{k}}{\partial p_{1}} \\
-\tilde{F}_{\mathrm{cc}}\left(\tilde{s}_{k}+\frac{\partial \mathcal{F}_{k}}{\partial p_{2}}\right)
\end{array}\right]
$$

and $\boldsymbol{p}:=\left[\begin{array}{ll}p_{1} & p_{2}\end{array}\right]^{\top}$. Obviously the parameter $\boldsymbol{p}$ is able to estimate by an RLS filter or an RTLS filter. We simply refer to these methods as RLS and RTLS respectively in the following sections.

The pair of the term $Q_{\mathrm{cc}, k}$ and the term $I_{\mathrm{typ}} t_{k}$ is the main obstacle due to those unboundedness. A simple solution is a differential approach, that is an RLS filter or an RTLS filter based on the following relation:

$$
\Delta y_{k}=\boldsymbol{p}_{k}^{\top} \Delta \boldsymbol{u}_{k}
$$

where $\Delta y_{k}:=y_{k}-y_{k-1}, \Delta \boldsymbol{u}_{k}:=\boldsymbol{u}_{k}-\boldsymbol{u}_{k-1}$. We refer to these methods as DRLS and DRTLS.

Our approach is considering a time-dependent local coordinate system of the vector space to which the pair $\left(y_{k}, \boldsymbol{u}_{k}\right)$ is belonging. The local coordinate of the pair is able to be bounded, if the origin of the local coordinate system is set close to the pair.

Let $\mathcal{V}$ be a vector space to which the pair $\left(y_{k}, \boldsymbol{u}_{k}\right)$ is belonging, we regard $\mathcal{V}$ as an affine space. Let $\mathcal{T}_{k}$ be a tangent space of $\mathcal{V}$ whose origin is set on $\left(y_{k}, \boldsymbol{u}_{k}\right)$ and whose bases are same to those of the original vector space. Then the local coordinate of $\left(y_{l}, \boldsymbol{u}_{l}\right) \in \mathcal{V}$ is written as $\left(y_{l}-y_{k}, \boldsymbol{u}_{l}-\boldsymbol{u}_{k}\right)=$ : $\left(\phi_{k}^{-1}\left(y_{l}\right), \psi_{k}^{-1}\left(\boldsymbol{u}_{l}\right)\right)$ in $\mathcal{T}_{k}$ for all $l$.

Now we consider a weighted mean of $y_{k}$ and $\boldsymbol{u}_{k}$ defined as follows:

$$
\bar{y}_{k}:=\frac{1}{S_{k}} \sum_{l=0}^{k} \lambda^{k-l} y_{l}, \quad \overline{\boldsymbol{u}}_{k}:=\frac{1}{S_{k}} \sum_{l=0}^{k} \lambda^{k-l} \boldsymbol{u}_{l}
$$

where $S_{k}:=\sum_{l=0}^{k} \lambda^{k-l}$ and $\lambda$ is a forgetting factor in $(0,1)$. Then the following relationship holds between the deviations of $y_{l}$ and $\boldsymbol{u}_{l}$ from the weighted mean: $y_{l}-\bar{y}_{k}=\boldsymbol{p}^{\top}\left(\boldsymbol{u}_{l}-\overline{\boldsymbol{u}}_{k}\right)$.

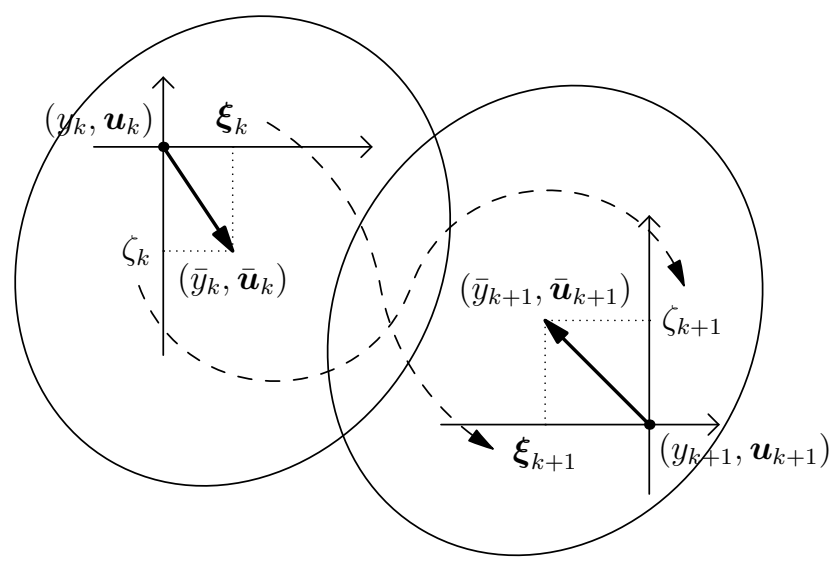

Fig. 3. Geometric relationship among the tangent spaces $\mathcal{T}_{k}$, the samples $\left(y_{k}, \boldsymbol{u}_{k}\right)$ and the weighted mean $\left(\bar{y}_{k}, \overline{\boldsymbol{u}}_{k}\right)$. The ellipses express the distributions of the samples. The equations (20) and (21) are the direct calculation from $\left(\zeta_{k}, \boldsymbol{\xi}_{k}\right)$ to $\left(\zeta_{k+1}, \boldsymbol{\xi}_{k+1}\right)$. Remark the difference between the two distributions is extremely emphasized.

The relationship also holds on the tangent space $\mathcal{T}_{k}$, because the map $\phi_{k}$ and $\psi_{k}$ conserves the inner product of $\mathcal{V}$. Therefore

$$
\zeta_{k}=\boldsymbol{p}^{\top} \boldsymbol{\xi}_{k},
$$

where $\left(\zeta_{k}, \boldsymbol{\xi}_{k}\right):=\left(\phi_{k}^{-1}\left(\bar{y}_{k}\right), \psi_{k}^{-1}\left(\overline{\boldsymbol{u}}_{k}\right)\right)$ (see Fig. 3).

The local coordinate of the weighted means $\zeta_{k}, \xi_{k}$ are calculated recursively by follows:

$$
\begin{aligned}
\zeta_{k} & =\phi_{k}^{-1}\left(\lambda S_{k-1} \phi_{k-1}\left(\zeta_{k-1}\right)+y_{k}\right) / S_{k} \\
& =\frac{\lambda S_{k-1}}{S_{k}}\left(\zeta_{k-1}-\Delta y_{k}\right), \\
\boldsymbol{\xi}_{k} & =\psi_{k}^{-1}\left(\lambda S_{k-1} \psi_{k-1}\left(\boldsymbol{\xi}_{k-1}\right)+y_{k}\right) / S_{k} \\
& =\frac{\lambda S_{k-1}}{S_{k}}\left(\boldsymbol{\xi}_{k-1}-\Delta \boldsymbol{u}_{k}\right) .
\end{aligned}
$$

The variables $\Delta y_{k}$ and $\Delta \boldsymbol{u}_{k}$ are bounded if $y_{k}$ and $\boldsymbol{u}_{k}$ are smooth time series, and calculated without dealing with the term $q_{\mathrm{cc}, k}$ and $t_{k}$, by using following equations: $t_{k}-t_{k-1}=$ $t_{s}, q_{\mathrm{cc}, k}-q_{\mathrm{cc}, k-1}=t_{s} I_{k-1}$. The term $S_{k}$ is also bounded obviously, and calculated recursively by $S_{k}=S_{k-1}+\lambda^{k}$ and $\lambda^{k}=\lambda \cdot \lambda^{k-1}$. Therefore $\zeta_{k}$ and $\boldsymbol{\xi}_{k}$ are bounded for each $k$, because $\lambda S_{k} / S_{k-1}<1$.

An adaptive filter based on (17) or (19) is more desirable for implementation in embedded systems than that based on (16), because all variables in the recursive calculation are bounded.

\section{Rayleigh quotient-based fast RTLS filter}

Let $\delta_{k}$ be the error of $\zeta_{k}$ and $\boldsymbol{\epsilon}_{k}$ be the error of $\boldsymbol{\xi}_{k}$, an RTLS filter minimizes the following objective function:

$$
J_{k}\left(\boldsymbol{p}, \hat{\mathcal{Z}}_{k}, \hat{\Xi}_{k}\right):=\sum_{l=0}^{k} \lambda^{k-l}\left(\delta_{l}^{2}+\boldsymbol{\epsilon}_{l}^{\top} W^{-1} \boldsymbol{\epsilon}_{l}\right),
$$

such that $\zeta_{k}-\delta_{k}=\boldsymbol{p}^{\top}\left(\boldsymbol{\xi}_{k}-\boldsymbol{\epsilon}_{k}\right)$, where $\hat{\mathcal{Z}}_{k}:=\left\{\hat{\zeta}_{0}, \ldots, \hat{\zeta}_{k}\right\}$, $\hat{\Xi}_{k}:=\left\{\hat{\boldsymbol{\xi}}_{0}, \ldots, \hat{\boldsymbol{\xi}}_{k}\right\}, \hat{\zeta}_{k}:=\zeta_{k}-\delta_{k}, \hat{\boldsymbol{\xi}}_{k}:=\boldsymbol{\xi}_{k}-\boldsymbol{\epsilon}_{k}$ and $W$ is a symmetric positive definite weighting matrix. Let $\hat{\boldsymbol{p}}_{k}$ be an 
estimator of $\boldsymbol{p}$ at $t_{k}$, the minimum point of $\left(\hat{\mathcal{Z}}_{k}, \hat{\Xi}_{k}\right)$ for each $\hat{\boldsymbol{p}}_{k}$ is given by:

$$
\hat{\boldsymbol{\eta}}_{k}:=A_{k}\left(A_{k}^{\top} \Lambda^{-1} A_{k}\right)^{-1} A_{k}^{\top} \Lambda^{-1} \boldsymbol{\eta}_{k},
$$

where

$$
\hat{\boldsymbol{\eta}}_{k}:=\left[\begin{array}{l}
\hat{\zeta}_{k} \\
\hat{\boldsymbol{\xi}}_{k}
\end{array}\right], \quad \boldsymbol{\eta}_{k}:=\left[\begin{array}{c}
\zeta_{k} \\
\boldsymbol{\xi}_{k}
\end{array}\right], \quad A_{k}:=\left[\begin{array}{c}
\hat{\boldsymbol{p}}_{k}^{\top} \\
I
\end{array}\right]
$$

and $\Lambda:=\operatorname{diag}\{1, W\}$.

A nonzero vector $\boldsymbol{a}_{k}$ such that $A_{k}^{\top} \boldsymbol{a}_{k}=0$ exists uniquely except for scalar multiplies, because the kernel of $A_{k}$ is an 1-dimensional subspace. Then minimizing objective function $J_{k}$ is equivalent to minimizing a Rayleigh quotient defined as follows:

$$
Q_{k}:=\frac{\boldsymbol{a}_{k}^{\top} \bar{R}_{k} \boldsymbol{a}_{k}}{\boldsymbol{a}_{k}^{\top} \Lambda \boldsymbol{a}_{k}}, \quad \text { where } \quad \bar{R}_{k}:=\sum_{l=0}^{k} \lambda^{k-l} \boldsymbol{\eta}_{l} \boldsymbol{\eta}_{l}^{\top} .
$$

The vector $\boldsymbol{a}_{k}$ must be parameterized as $\boldsymbol{a}_{k}^{\top}=\left[\begin{array}{ll}-1 & \boldsymbol{p}_{k}^{\top}\end{array}\right]$ to satisfy the orthogonality condition. Now we consider the following incremental update equation:

$$
\hat{\boldsymbol{p}}_{k}=\hat{\boldsymbol{p}}_{k-1}+\theta_{k} \boldsymbol{w}_{k},
$$

where $\boldsymbol{w}_{k}$ is a direction of a line search. The direction $\boldsymbol{w}_{k}$ is desirably a time series of vectors which efficiently spans the image of $A_{k}$ in a short time range. Although authors employ $\boldsymbol{\xi}_{k}$ as $\boldsymbol{w}_{k}$ in [10], we employ a random unit vector uniformly distributed on the unit circle (actually it is not necessarily normalized), because $\boldsymbol{\xi}_{k}$ is strongly time-correlated and inefficient to span the image of $A_{k}$ in our case.

Substituting (25) into (24), the Rayleigh quotient $Q_{k}$ forms a rational function of $\theta_{k}$. The numerator $N_{k}$ and the denominator $D_{k}$ of $Q_{k}$ is written as

$$
\begin{aligned}
& N_{k}=N_{2, k} \theta_{k}^{2}+2 N_{1, k} \theta_{k}+N_{0, k}, \\
& D_{k}=D_{2, k} \theta_{k}^{2}+2 D_{1, k} \theta_{k}+D_{0, k},
\end{aligned}
$$

where

$$
\begin{aligned}
& N_{2, k}:=\boldsymbol{w}_{k}^{\top} R_{k} \boldsymbol{w}_{k}, \\
& N_{1, k}:=\boldsymbol{w}_{k}^{\top} R_{k} \hat{\boldsymbol{p}}_{k-1}-\boldsymbol{c}_{k}^{\top} \boldsymbol{w}_{k}, \\
& N_{0, k}:=\hat{\boldsymbol{p}}_{k-1}^{\top} R_{k} \hat{\boldsymbol{p}}_{k-1}-2 \boldsymbol{c}_{k}^{\top} \hat{\boldsymbol{p}}_{k-1}+d \\
& D_{2, k}:=\boldsymbol{w}_{k}^{\top} W \boldsymbol{w}_{k}, \\
& D_{1, k}:=\boldsymbol{w}_{k}^{\top} W \hat{\boldsymbol{p}}_{k-1} \\
& D_{0, k}:=\hat{\boldsymbol{p}}_{k-1}^{\top} W \hat{\boldsymbol{p}}_{k-1}+1
\end{aligned}
$$

\begin{tabular}{|c|c|c|c|c|c|c|c|}
\hline$\theta_{k}$ & $-\infty$ & $\cdots$ & $\theta_{k}^{+}$ & $\cdots$ & $\theta_{k}^{-}$ & $\ldots$ & $\infty$ \\
\hline$\partial Q_{k} / \partial \theta_{k}$ & 0 & - & 0 & + & 0 & - & 0 \\
\hline$Q_{k}$ & $*$ & $\searrow$ & & $\nearrow$ & & $\searrow$ & * \\
\hline
\end{tabular}

and

$$
\left[\begin{array}{ll}
d_{k} & \boldsymbol{c}_{k}^{\top} \\
\boldsymbol{c}_{k} & R_{k}
\end{array}\right]:=\bar{R}_{k}=\sum_{l=0}^{k} \lambda^{k-l}\left[\begin{array}{cc}
\zeta_{k}^{2} & \zeta_{k} \boldsymbol{\xi}_{k}^{\top} \\
\boldsymbol{\xi}_{k} \zeta_{k} & \boldsymbol{\xi}_{k} \boldsymbol{\xi}_{k}^{\top}
\end{array}\right] .
$$

The extreme points of $Q_{k}$ are given by the solutions of following equation

$$
\frac{\frac{\partial N_{k}}{\partial \theta_{k}} D_{k}-N_{k} \frac{\partial D_{k}}{\partial \theta_{k}}}{D_{k}^{2}}=\frac{\alpha_{k} \theta_{k}^{2}+\beta_{k} \theta_{k}+\gamma_{k}}{D_{k}^{2} / 2}=0,
$$

TABLE I. THE FIRST DERIVATIVE TEST OF THE RAYLEIGH QUOTIENT

\begin{tabular}{|c||c|c|c|c|c|c|c|}
\multicolumn{1}{|c}{ For $\alpha_{k}>0$} \\
\hline$\theta_{k}$ & $-\infty$ & $\cdots$ & $\theta_{k}^{-}$ & $\cdots$ & $\theta_{k}^{+}$ & $\cdots$ & $\infty$ \\
\hline$\partial Q_{k} / \partial \theta_{k}$ & 0 & + & 0 & - & 0 & + & 0 \\
\hline$Q_{k}$ & $* 1$ & $\nearrow$ & & $\searrow$ & & $\nearrow$ & $*$ \\
\hline
\end{tabular}

For $\alpha_{k}<0$

TABLE II. BATTERY MODEL PARAMETERS USED IN NUMERICAL EVALUATION

\begin{tabular}{|cc||cr|}
\hline Parameter & Value & Parameter & Value \\
\hline \hline$R_{0}$ & $10 \mathrm{~m} \Omega$ & $\tilde{F}_{\mathrm{cc}}$ & $1.0 \mathrm{Ah}$ \\
\hline$R_{d}$ & $1.0 \mathrm{~m} \Omega$ & $E_{0}$ & $2.6 \mathrm{~V}$ \\
\hline$C_{d}$ & $5.0 \mathrm{kF}$ & $E_{1}$ & $1.6 \mathrm{~V}$ \\
\hline
\end{tabular}

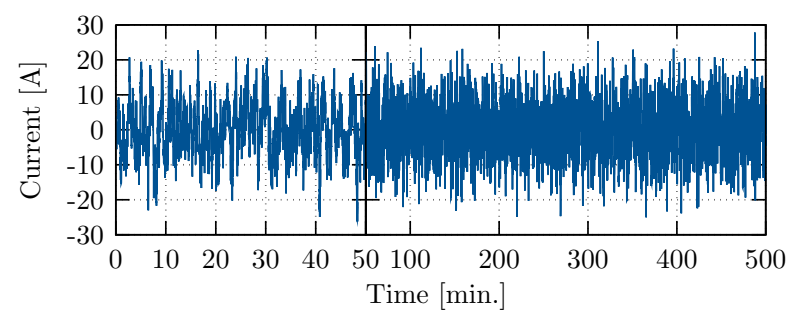

Fig. 4. Input current for the simulation. The time axis is zoomed in during the first 50 minutes.

where

$$
\begin{aligned}
\alpha_{k} & :=N_{2, k} D_{1, k}-N_{1, k} D_{2, k}, \\
\beta_{k} & :=N_{2, k} D_{0, k}-N_{0, k} D_{2, k}, \\
\gamma_{k} & :=N_{1, k} D_{0, k}-N_{0, k} D_{1, k} .
\end{aligned}
$$

Then $\theta_{k}$ should be a root of the quadratic form of the numerator of (28). From the first derivative test of $Q_{k}$ shown in Table I, the quadratic form has two distinct roots for nonzero $a_{k}$, and the minimum point of $Q_{k}$ is given by $\theta_{k}^{+}$, where

$$
\theta_{k}^{ \pm}:=\frac{-\beta_{k} \pm \sqrt{\beta_{k}^{2}-4 \alpha_{k} \gamma_{k}}}{2 \alpha_{k}} .
$$

When the $a_{k}$ is incidentally close to zero, we employ $\theta_{k}=0$ to avoid numerical instability.

\section{NumERICAL EXAMPLE}

In this section, we illustrate the performance of our algorithm by a numerical simulation. In our simulation, we employed the simplified battery model shown in Fig. 2 with the battery model parameters in Table II, and sampling period $t_{s}=100 \mathrm{~ms}$. We assumed the dependency of the voltage source $E$ on the state of charge $s$ is described as $E=E_{0}+E_{1} s$, where $E_{0}$ and $E_{1}$ are constants.

First, we calculated the terminal voltage of the battery using the input current shown in Fig. 4 (the true SoC is also shown in Fig. 5 for visibility). We slightly varied the FCC of the battery from the typical value as $F_{\mathrm{cc}}=0.9 \tilde{F}_{\mathrm{cc}}$. 


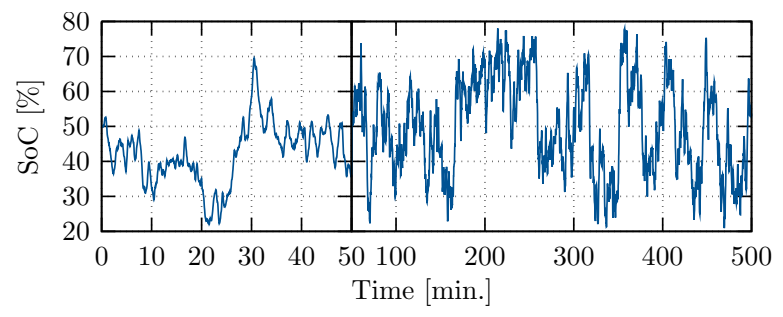

Fig. 5. State of charge simulated from the input current shown in Fig. 4. The full charge capacity $F_{\mathrm{cc}}$ is assumed to be $1.0 \mathrm{Ah}$.

TABLE III. VARIATION OF EVALUATED ALGORITHMS

\begin{tabular}{|c|c|c|c|}
\hline & Basic & Differential & Affine \\
\hline RLS & RLS & DRLS & ARLS \\
RTLS & TLS & DTLS & ATLS \\
\hline
\end{tabular}

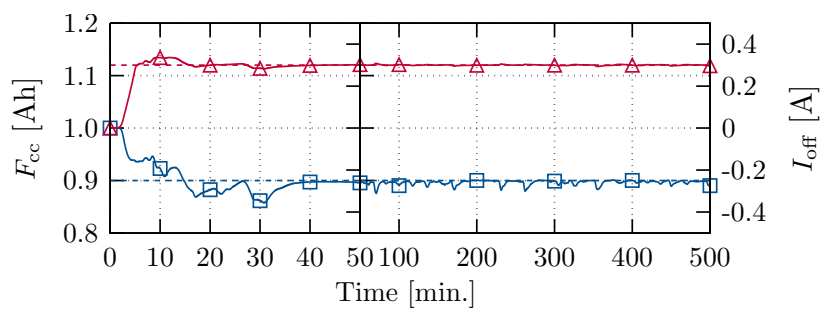

Fig. 6. Estimated $F_{\mathrm{cc}}$ (square) and $I_{\text {off }}$ (triangle) by RLS.

Next, we estimated the SoC of the battery and the derivative thereof from the current and the voltage using a Kalman filter based on the simplified battery model, where we offset the current by $0.3 \mathrm{~A}$, and added a Gaussian noise of variance $\sigma_{I}^{2}=10^{-4}$ and $\sigma_{V}^{2}=10^{-5}$ to the current and the voltage respectively.

After that, we estimated the FCC and the current sensor offset from the estimated SoC, the derivative thereof and the measured current (or Coulomb counting in some methods) by various adaptive filters shown in Table III. The differential filters are based on (17), while the basic and affine algorithms are based on (16) and (19) respectively.

We employed $\lambda=e^{-\tau / t_{s}}$ where $\tau=20 \mathrm{~min}, I_{\mathrm{typ}}=100 \mathrm{~A}$ and $W=I$. Then we tuned the intial $R_{k}, \boldsymbol{c}_{k}, d_{k}$ as far as possible, so that the estimated values converge while $t_{k} \leq$ $100 \mathrm{~min}$.

The results of the estimation are shown in Fig. 6-11. All filters well estimate the current sensor offset, and well estimate the FCC except RTLS. The estimation errors, means and standard deviations thereof are shown in Fig. 12-15. ARLS and ARTLS achieve equivalent accuracy to that of RLS, while the results of DRLS and DRTLS are biased in $F_{\mathrm{cc}}$ and have larger deviations in $I_{\text {off }}$.

\section{CONClusion}

In this paper, we propose a method to estimate the FCC of a lithium-ion battery by an adaptive filter from the current and the $\mathrm{SoC}$ of the battery. The $\mathrm{SoC}$ is estimated by a Kalman filter based on an simplified battery model from the current and the terminal voltage of the battery. The bias error of the estimated SoC caused by the current sensor offset and the difference between a true value of the FCC and a typical value thereof

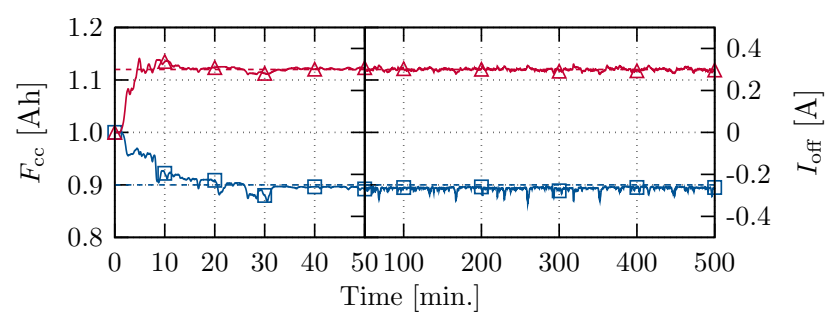

Fig. 7. Estimated $F_{\mathrm{cc}}$ (square) and $I_{\mathrm{off}}$ (triangle) by DRLS.

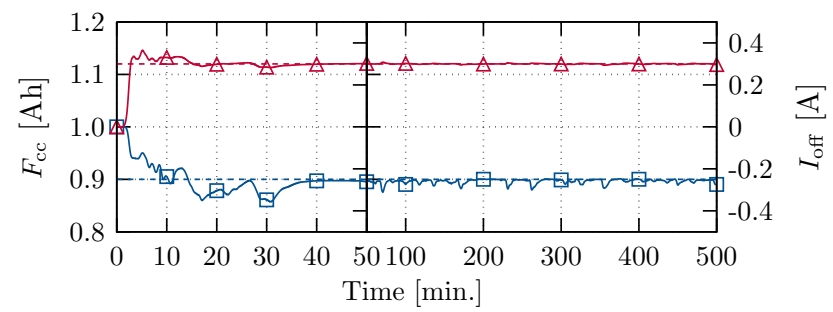

Fig. 8. Estimated $F_{\mathrm{cc}}$ (square) and $I_{\mathrm{off}}$ (triangle) by ARLS.

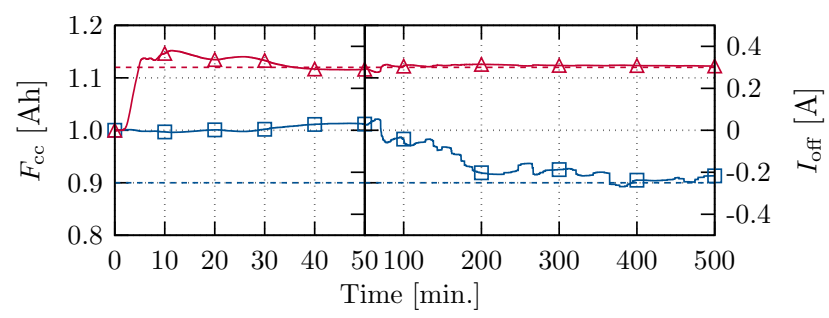

Fig. 9. Estimated $F_{\mathrm{cc}}$ (square) and $I_{\mathrm{off}}$ (triangle) by RTLS.

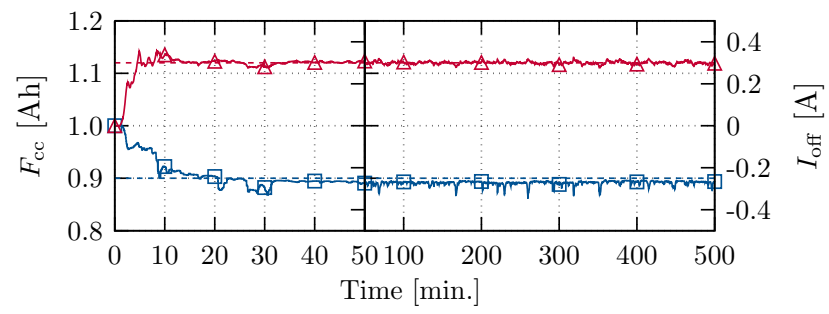

Fig. 10. Estimated $F_{\mathrm{cc}}$ (square) and $I_{\mathrm{off}}$ (triangle) by DRTLS.

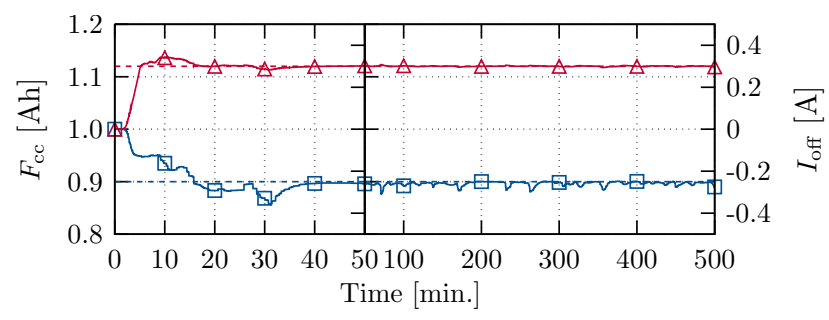

Fig. 11. Estimated $F_{\mathrm{cc}}$ (square) and $I_{\mathrm{off}}$ (triangle) by ARTLS.

assumed in the Kalman filter is compensated in the meaning of first order approximation.

In the FCC estimation by commonly used RLS filter or RTLS filter where the current sensor offset is taken into consideration, the variables used in the estimation algorithms are unbounded. In our approach, the RLS filter or RTLS filter is composed on an affine space to avoid the unboundedness, instead of differentiating the input signals, which is another 


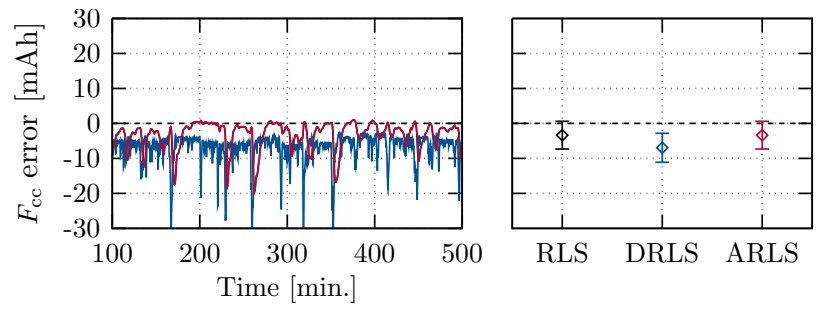

Fig. 12. Estimation error of $F_{\mathrm{cc}}$ by RLS, DRLS and ARLS, and means and standard deviations of the errors from $100 \mathrm{~min}$. to $500 \mathrm{~min}$.
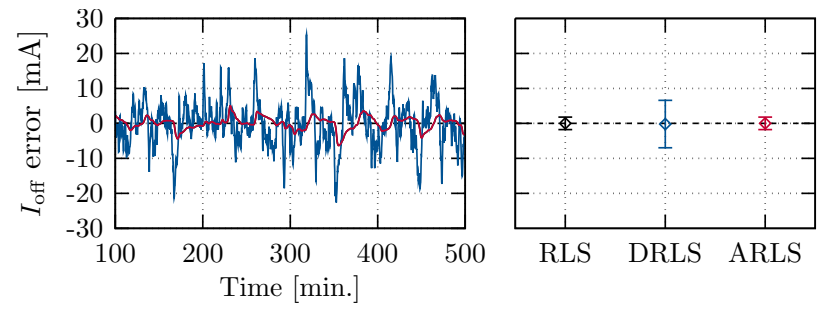

Fig. 13. Estimation error of $I_{\text {off }}$ by RLS, DRLS and ARLS, and means and standard deviations of the errors from $100 \mathrm{~min}$. to $500 \mathrm{~min}$.
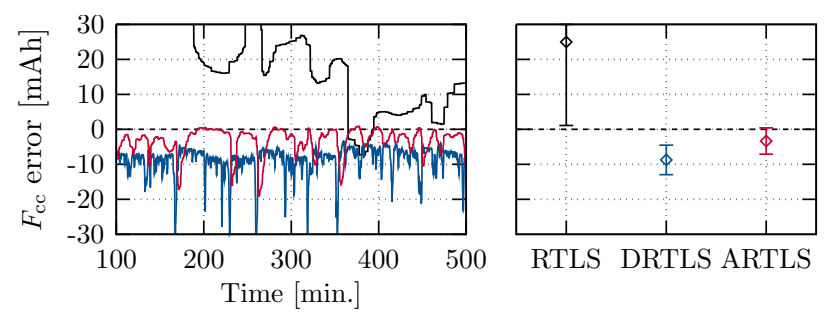

RTLS DRTLS ARTLS

Fig. 14. Estimation error of $F_{\mathrm{cc}}$ by RTLS, DRTLS and ARTLS, and means and standard deviations of the errors from $100 \mathrm{~min}$. to $500 \mathrm{~min}$.
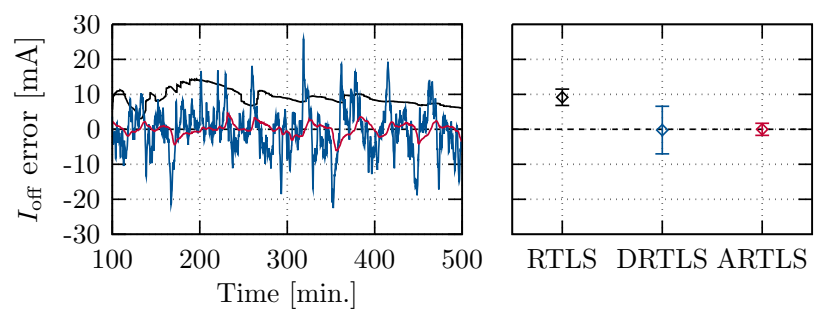

Fig. 15. Estimation error of $I_{\text {off }}$ by RTLS, DRTLS and ARTLS, and means and standard deviations of the errors from $100 \mathrm{~min}$. to $500 \mathrm{~min}$.

approach to keep the variables bounded. We illustrate that our approach outperforms the differentiation approach by a numerical example.

\section{REFERENCES}

[1] G. Pistoia, "Portable devices: Batteries," in Encyclopedia of Electrochemical Power Sources, J. Garche, Ed. Elsevier, 2009, pp. 29-38.

[2] L. Gao, S. Liu, and R. A. Dougal, "Dynamic lithium-ion battery model for system simulation," IEEE Trans. Compon. Packag. Technol., vol. 25, no. 3, pp. 495-505, 2002.

[3] L. Lu, X. Han, J. Li, J. Hua, and M. Ouyang, "A review on the key issues for lithium-ion battery management in electric vehicles," J. Power Sources, vol. 226, pp. 272-288, 2013.

[4] J. Lee, O. Nam, and B. H. Cho, "Li-ion battery SoC estimation method based on the reduced order extended Kalman filtering," J. Power Sources, vol. 174, pp. 9-15, 2007.

[5] H. Rahimi-Eichi and M.-Y. Chow, "Adaptive parameter identification and state-of-charge estimation of lithium-ion batteries," in 38th Annual Conference on IEEE Industrial Electronics Society (IECON), 2012, pp. 4012-4017.

[6] S. Fengchun, H. Xiaosong, Z. Yuan, and L. Siguang, "Adaptive unscented Kalman filtering for state of charge estimation of a lithium-ion battery for electric vehicles," Energy, vol. 36, pp. 3531-3540, 2011.

[7] G. L. Plett, "Recursive approximate weighted total least squares estimation of battery cell total capacity," J. Power Sources, vol. 196, no. 4, pp. 2319-2331, 2011.

[8] _ _ "Extended Kalman filtering for battery management systems of LiPB-based HEV battery packs Part 2. Modeling and identification," $J$. Power Sources, vol. 134, pp. 262-276, 2004.

[9] D. Andrea, Battery Management Systems for Large Lithium-ion Battery Packs. Artech house, 2010.

[10] D.-Z. Feng, X.-D. Zhang, D.-X. Chang, and W. X. Zheng, "A fast recursive total least squares algorithm for adaptive FIR filtering," vol. 52, no. 10, pp. 2729-2737, 2004.

[11] J. Yamaki, "Secondary batteries - lithium rechargeable systems lithium-ion - overview," in Encyclopedia of Electrochemical Power Sources, J. Garche, Ed. Elsevier, 2009, pp. 183-191.

[12] R. Alcántara, P. Lavela, J. L. Tirado, E. Zhecheva, and R. Stoyanova, "Recent advances in the study of layered lithium transition metal oxides and their application as intercalation electrodes," J. Solid State Electrochem., vol. 3, no. 3, pp. 121-134, 1999.

[13] V. R. Subramanian, V. Boovaragavan, V. Ramadesigan, and M. Arabandi, "Mathematical model reformulation for lithium-ion battery simulations: Galvanostatic boundary conditions," J. Electrochem. Soc., vol. 156, no. 4, pp. A260-A271, 2009.

[14] A. Baba and S. Adachi, "State of charge estimation of lithium-ion battery using Kalman filters," in IEEE International Conference on Control Applications (CCA), 2012, pp. 409-414.

[15] M. Doyle, T. F. Fuller, and J. Newman, "Modeling of galvanostatic charge and discharge of the lithium/polymer/insertion cell," J. Electrochem. Soc., vol. 140, no. 6, pp. 1526-1533, 1993.

[16] D. A. Harville, Matrix Algebra From a Statistician's Perspective. Springer, 2008. 